\title{
Habilidades teóricas y prácticas para flebotomía entre alumnos de primer semestre de medicina en el Centro de Simulación Montagne, Mérida - México
}

\section{Theoretical and practical flebotomy skills among first-semester medicine students at the Montagne Simulation Center, Mérida - México}

\author{
Gregorio Cetina-Sauri ${ }^{1}$, Herbert Puga-Matú ${ }^{2}$, Jaidy Chávez-Medina ${ }^{3}$, Joeana Cambranis-Romero ${ }^{4}$, \\ Esteban Aguilar-Vargas ${ }^{5}$, Nina Méndez-Domínguez ${ }^{6}$ \\ ${ }^{1}$ Médico especialista en pediatria, responsable del Centro de Simulación Médica Montagne. Universidad Marista de Mérida, Mérida, México. \\ ${ }^{2}$ Médico cirujano, profesor de la licenciatura en Medicina. Universidad Marista de Mérida, Mérida, México. \\ ${ }^{3}$ Química farmacobióloga, responsable del laboratorio de fisiologia. Universidad Marista de Mérida, Mérida, México. \\ ${ }^{4}$ Ingeniera biomédica, profesora adjunta. Universidad Marista de Mérida, Mérida, México. \\ ${ }^{5}$ Médico Pasante en Servicio Social, Mérida, México. \\ ${ }^{6}$ Médico cirujano, Doctora en Ciencias de la Salud. Profesora de tiempo completo en el Campus de Ciencias de la Salud, Mérida, México.
}

Correspondencia

Nina Méndez-Domínguez

Teléf. (999) 942-9700.

Direcc. Universidad Marista de

Mérida. Periférico Norte Tablaje

Catastral 13941 Carretera Mérida

- Progreso. C.P. 97300 Mérida

Yucatán, México.

nmendez@marista.edu.mx

Recibido: 3 de enero 2017.

Aceptado: 7 de agosto 2017.

Financiamiento: Autofinanciado.

Conflictos de interés: Los autores declaran no tener conflictos de interés.

Citar como: Cetina-Sauri G, Puga-Matú H, Chávez-Medina J, Cambranis-Romero J, AguilarVargas E, Méndez-Domínguez N. Habilidades teóricas y prácticas para flebotomía entre alumnos de primer semestre de medicina en el Centro de Simulación Montagne, Mérida - México. An Fac med. 2017;78(4):414-8

DOI: http://dx.doi.org/10.15381/ anales.v78i4.14263
An Fac med. 2017;78(4):414-8 / http://dx.doi.org/10.15381/anales.v78i4.14263

\section{Resumen}

Introducción. El uso de simuladores de pacientes humanos es una estrategia eficaz en la enseñanza de procedimientos clínicos. Sin embargo, existe poca información para ayudar a los profesores a mejorar sus estrategias docentes permitiéndoles mejorar las experiencias de aprendizaje de acuerdo a la edad o el sexo de los alumnos que emplean este tipo de simulación. Objetivos. Identificar si existen diferencias por sexo 0 edad en asociación a las habilidades de los alumnos del primer semestre de medicina para la flebotomía. Diseño. Cuasiexperimental. Lugar. Centro de Simulación Médica Montagne de la Universidad Marista de Mérida. Participantes. Estudiantes de primer semestre de la licenciatura en medicina. Intervenciones. Intervención educativa del proceso de flebotomía con toma de muestra, empleando simuladores de alta fidelidad. Para el análisis, se evaluó la asociación de las variables de edad y sexo con el desempeño de los alumnos. Principales medidas de los resultados. Se analizaron las evaluaciones prácticas y teóricas. Para la evaluación práctica se aplicó una rúbrica de 20 ítems y se promediaron las puntuaciones de las esferas teóricas y prácticas de los alumnos. Resultados. Se identificó que las mujeres desempeñaron mejor sus habilidades prácticas $(p<0,03)$ aún en el modelo de regresión lineal ajustado por edad y grupo. Discusión. El mejor desempeño en la práctica de flebotomía entre las alumnas de medicina difícilmente sea un hallazgo aislado, pues puede derivarse de las habilidades para la comunicación interpersonal, misma que también puede ser reforzada en los alumnos del sexo masculino.

Palabras Clave. Educación de Pregrado en Medicina; Enseñanza Mediante Simulación de Alta Fidelidad; Flebotomía.

\section{Abstract}

Introduction. The use of human patient simulators is an effective strategy in the teaching of clinical procedures. However, there is little information to help teachers improve their teaching strategies, allowing them to improve learning experiences according to the age or sex of students who use this type of simulation. Objectives. To identify if there are differences by sex or age in association with the skills of the students of the first semester of medicine for phlebotomy. Design. Quasiexperimental. Places. Medical Simulation Centre Montagne of the Marista University of Merida. Participants. First semester medical students. Interventions. Educational intervention of the phlebotomy process with sample taking, and using high fidelity simulators. For the analysis, sociodemographic variables were associated with the performance of the students. Main result measures. The practical and theoretical evaluations were analyzed. For the practical evaluation, a rubric of 20 items was applied and the scores of the theoretical and practical areas of the students were averaged. Results. It was identified that women performed their practical skills better $(p<0.03)$ even in the linear regression model adjusted for age and group. Discussion. The best performance in the practice of phlebotomy among medical students is hardly an isolated finding, since they could derive from the skills for interpersonal communication, which can also be reinforced in male students.

Keywords. Education Medical Undergraduate; High Fidelity Simulation Training; Phlebotomy. 


\section{INTRODUCCIÓN}

La simulación médica de alta fidelidad ha permitido que la educación médica vincule los aspectos teóricos a los prácticos desde muy temprano en la formación de pregrado en medicina, ya que, a diferencia de tiempos pasados, las prácticas de los alumnos no implican un riesgo para el paciente y sí le permiten familiarizarse con el trato al paciente y sus familiares e ir desarrollando habilidades clínicas de utilidad ${ }^{(1,2)}$.

El uso de simuladores de pacientes humanos es una estrategia de enseñanza eficaz en muchas áreas de la salud. Pero existe poca información para ayudar a los profesores a mejorar las estrategias docentes que implican el empleo de este tipo de simulación. Por ello, conocer si las características individuales como lo son el sexo o la edad influencian sobre su desempeño practico o aprendizaje conceptual les permite implementar estrategias para optimizar el desempeño de los alumnos ${ }^{(3,4,5)}$.

Previamente se ha identificado que aspectos como el sexo y la edad pueden reflejarse en el desempeño de los alumnos a lo largo de su formación, por lo cual consideramos importante el hecho de identificar si estos aspectos inherentes a los alumnos se asocian a diferencias en el aprendizaje teórico o práctico de los alumnos de medicina, ya que podrían ayudarnos a comprender y optimizar entre ellos la generación de competencias médicas teóricas y prácticas ${ }^{(6)}$

Este artículo tiene como finalidad explorar si las características de los alumnos, en particular las diferencias en edad o sexo se asocian a diferencias en las competencias teóricas y prácticas de la flebotomía con toma de muestra de acuerdo a lo evaluado en el Centro de Simulación (CS) Montagne en alumnos de primer semestre de medicina.

\section{MÉTODOS}

Se incluyó en el presente estudio a la totalidad de alumnos regulares de la generación 2016, quienes organizados en cuatro grupos fueron evaluados en las esferas tanto teóricas como prácticas. La evaluación escrita fue administrada en la tercera sesión del semestre y la práctica, durante la cuarta en todos los grupos.

Mediante un examen escrito con diez reactivos de opción múltiple con elementos gráficos se abordaron los aspectos teóricos inherentes a la realización de la flebotomía con toma de muestra. Antes de su aplicación, el examen fue realizado y revisado por expertos en cuanto a los elementos de compresión, claridad, validez de contenido y constructo.

Debido a que la formación médica básica en la Universidad Marista de Mérida implica no solo el aprendizaje de los aspectos teórico prácticos, sino también la formalidad, actitud, presentación y trato interpersonal ético y respetuoso (denominado el ser para servir), la rúbrica de evaluación de la práctica de flebotomía incluye aspectos directa e indirectamente relacionados con el procedimiento de flebotomía, mismos que se detallan a continuación:

Comunicación con el paciente. En cuanto al trato interpersonal respetuoso y ético asociado a la flebotomía, la rúbrica evalúa que los alumnos pongan en práctica los principios éticos al presentarse ante el paciente, llevar a cabo el procedimiento de consentimiento informado explicando el procedimiento mencionando objetivos, alcances y potenciales efectos no deseados. Posteriormente, el alumno debe solicitar el consentimiento del paciente, proporcionarle indicaciones precisas, proceder a la flebotomía con toma de muestra y al finalizar, no debe olvidar despedirse del paciente.

Asepsia e higiene. Las medidas correctas de asepsia y antisepsia son un factor imprescindible durante una flebotomía y en general para la práctica clínica, por ello, en la práctica de flebotomía con toma de muestra se evalúa que el alumno realice correctamente el lavado de manos, la colocación de los guantes, la asepsia del área de punción, el manejo estéril del material, así como que el alumno retire el material empleado y limpie el espacio empleado.

Técnica. Los aspectos específicos de la técnica de flebotomía que se evalúan durante la práctica incluyen la selección, revisión y preparación del material, posicionamiento del brazo del paciente, la elección de la vena más adecuada, colocación de la ligadura, la preparación de la jeringa, seguida de la introducción en el ángulo correcto de la aguja, extracción de la sangre necesaria y el soltar la ligadura oportunamente, finalizando con la extracción la aguja y reposicionamiento del brazo.

Retroalimentación e integración de la calificación final. Los alumnos fueron evaluados con la rúbrica y recibieron una retroalimentación constructiva posterior a su práctica, en la cual se les indicaron los sus fortalezas y oportunidades de mejora de manera específica, adicionalmente les fueron proyectadas las videograbaciones con las cuales pudieron analizar críticamente su desempeño. Los alumnos tuvieron la oportunidad de repetir sus prácticas y para efecto de este estudio se incluyó la calificación de la última práctica. Cada ítem de la rúbrica recibió un puntaje individual y la suma de todos los ítems constituyó la calificación. Para integrar la calificación final se estableció una ponderación de $50 \%$ para la evaluación práctica y $50 \%$ para la escrita.

Para efectos de evaluar las tendencias y distribución de la edad por sexo entre los alumnos, se obtuvo la estadística descriptiva y se desarrollaron análisis comparativos inter e intra-grupos, adicionalmente, se comparó la proporción por sexo y el promedio general de hombres y mujeres en la generación 2016 y se comparó con la de toda la licenciatura.

La variable dependiente fue el puntaje obtenido en la actividad, tanto en la esfera práctica, como en la escrita y el promedio de ambas. Las variables independientes fueron: la edad, como variable numérica continua; el sexo como variable binomial, tomando en cuenta al masculino (por su mayor frecuencia) como referencia y finalmente se incluyó al grupo (o salón) como una variable multinominal. Los datos fueron analizados con el software estadístico Stata $12^{\circ}$. Las pruebas de comparación de grupos que se realizaron fueron la prueba $t$ de student (pareadas o de dos grupos, según el caso) para comparación de medias em- 
pleando variables continuas y la prueba de $X^{2}$ se empleó para la comparación de proporciones entre grupos. Finalmente, se desarrolló análisis ad hoc en el cual se incluyeron las variables explicativas asegurando el ajuste entre ellas, empleando el método de máxima verosimilitud en un modelo de regresión lineal. El valor alfa para diferencias estadísticas se estableció en todos los análisis al 0,05.

\section{RESULTADOS}

La licenciatura en medicina de la Universidad Marista de Mérida cuenta con una matrícula total 437 alumnos de 21,80 $\pm 1,70$ años en promedio; el $51,50 \%$ son hombres y $48,50 \%$ mujeres; no existió diferencia en la edad media por sexo. A pesar de que el promedio general para los hombres fue de 82,06 y 82,10 para las mujeres, esta diferencia no fue significativa.

La generación 2016 inició con 138 alumnos matriculados, de los cuales, 75 $(54,35 \%)$ fueron hombres y $63(45,65 \%)$ mujeres, y tuvieron una edad media de $19,35 \pm 0,88$ años; la cual es equivalente en ambos sexos. La prueba chi $^{2}$ mostró que la proporción de alumnos del sexo masculino es mayor en los alumnos del primer semestre que en las demás generaciones. $(p<0,03)$.

Los alumnos que sustentaron la evaluación escrita de flebotomía( $\mathrm{N}=134)$ obtuvieron un puntaje de 86 en la evaluación teórica escrita, con un rango entre 60 y 100. Cuatro alumnos no sustentaron la prueba, por lo cual se excluyeron del análisis.

Posterior a la sesión práctica, se observó que los errores más frecuentes fueron relacionados con a) la explicación al paciente, b) la obtención del consentimiento informado, c) el retiro de la ligadura posterior a la toma de muestra $y$, por último, d) el despedirse del paciente, en orden de frecuencia y la generación obtuvo una puntuación de 81,5 en promedio $\pm 1,9$. Después de la retroalimentación, cuatro alumnos por cada grupo repitieron necesariamente la práctica mientras los demás lo realizaron para mejorar su desempeño. La evaluación fi-
Tabla 1. Porcentaje obtenido en la práctica de flebotomía en cada ítem ( $\mathrm{N}=134)$.

\begin{tabular}{|rlr}
\hline 1 & Puntual y uniforme completo & 95,24 \\
\hline 2 & Realiza asepsia de manos & 100,00 \\
\hline 3 & Se presenta con el paciente & 95,24 \\
\hline 4 & Informa del procedimiento & 95,24 \\
\hline 5 & Consentimiento informado & 81,43 \\
\hline 6 & Se coloca los guantes & 100,00 \\
\hline 7 & Revisa el equipo y los insumos & 85,71 \\
\hline 8 & Posiciona el brazo & 88,40 \\
\hline 9 & Realiza asepsia del área & 88,40 \\
\hline 10 & Coloca la ligadura & 100,00 \\
\hline 11 & Verifica posición de la vena & 85,71 \\
\hline 12 & Prepara jeringa & 100,00 \\
\hline 13 & Introduce la aguja & 100,00 \\
\hline 14 & Extrae sangre y suelta la ligadura & 61,90 \\
\hline 15 & Retira la jeringa & 85,71 \\
\hline 16 & Coloca la torunda en el brazo & 95,24 \\
\hline 17 & Da indicaciones al paciente & 81,43 \\
\hline 18 & Vierte el contenido de la jeringa & 85,71 \\
\hline 19 & Desecha correctamente el material & 76,19 \\
\hline 20 & Contaminado & 76,40 \\
\hline
\end{tabular}

nal desglosada por rubro se presenta en la tabla 1. Las calificaciones de las esferas prácticas, escritas y el promedio de las calificaciones se presentan en la tabla 2.

No se encontró asociación entre la edad y la puntuación obtenida por los alumnos, pero se identificaron diferencias asociadas al sexo, como se muestra en la tabla 3. Dichas diferencias permanecieron en el modelo de regresión lineal, aun después de ajustar por la edad y el grupo, tal como se puede apreciar en la tabla 4.

Tabla 2. Características de los alumnos y puntajes obtenidos por grupo $(\mathrm{N}=134)$.

\begin{tabular}{cccccc} 
Grupo & Práctica & Teórica & Promedio & Edad & \% Masculino \\
\hline A & 88 & 87 & 86,8 & 19,4 & 48,5 \\
B & 88 & 86 & 86,7 & 19,45 & 38,9 \\
C & 89 & 88 & 88,3 & 19,45 & 42,9 \\
D & 90 & 85 & 88,2 & 19,3 & 52,9 \\
\hline
\end{tabular}

\section{DISCUSIÓN}

Hemos mostrado que las puntuaciones obtenidas para flebotomía evaluadas mediante una rúbrica multidimensional muestran que las mujeres se desemesfera práctica a comparación de los varones, aún después de ajustar por factoedad. Las diferencias en las habilidades prácticas se reflejaron adicionalmente en la puntuación final promediada.

Aparte de lo común que fue la omisión de retirar la ligadura después de la toma de muestra, de acuerdo a las evaluaciones de la práctica, los puntos en los cuales se observó menor eficiencia masculina fueron aquellos relacionados con el trato interpersonal incluyendo la explicación al paciente, la obtención del consentimiento e incluso, el despedirse después del procedimiento, lo cual pudiera derivarse de que las mujeres con mayor frecuencia ser más empáticas con los pacientes. De acuerdo a un estudio previo, el nivel de empatía en residentes de medicina interna suele ser mayor en mujeres, teniendo su máxima expresión durante el primer año de la carrera, con una tendencia a decrecer año con año; dichos resultados son congruentes con los hallazgos posteriores que otros autores han identificado entre estudiantes de medicina de pregrado ${ }^{(7,8,9)}$.

En relación al efecto de la socialización y el trato interpersonal de los alumnos de medicina, existen estudios que han identificado que las mujeres son más propensas a elegir especialidades médicas que implican una socialización prolongada con los pacientes y que tienen preferencia por los aspectos sociales de la medicina, lo cual pudiera ser un reflejo de: peñaron significativamente mejor en la res como el grupo al que pertenecen y la 
Tabla 3. Calificaciones obtenidas en la práctica de flebotomía $(\mathrm{N}=134)$

\begin{tabular}{cccccc} 
Sexo & Media & \multicolumn{2}{c}{ Desviación estándar } & Intervalo Confianza 95\% \\
\hline Femenino & \multicolumn{5}{c}{ PROMEDIO } \\
Masculino & 88,54 & 5,31 & 87,29 & 89,79 \\
& 86,41 & 6,55 & 84,75 & 88,08 \\
\hline Femenino & 86,62 & 7,16 & TEÓRICO & 88,31 \\
Masculino & 85,57 & 7,19 & 84,92 & 87,41 \\
& & & 83,73 & \\
Femenino & 90,30 & 7,76 & PRÁCTICO & 98,47 & 92,15 \\
Masculino & 87,13 & 9,13 & 87,36 & 90,23 \\
\hline
\end{tabular}

a) el tipo de atención médica para la que se consideran más hábiles, b) que simplemente encuentran satisfacción en el trato interpersonal o c) una combinación de ambas. Cabe señalar que lo anterior no significa que las competencias medicas femeninas se limiten a las áreas sociales de la medicina, pues la información existente sugiere que la menor proporción de mujeres en la medicina tecnológicas o intervencionista no corresponde a una falta de habilidades, sino a las percepciones sociales asociadas al género ${ }^{(10,11,12)}$.

Entre las mujeres británicas que cursaban el primer año de medicina, se identificó que la comunicación interpersonal y la socialización son factores útiles que ayudan a las alumnas a enfrentar el estrés y sentimientos adversos, por lo cual, sería comprensible que las mujeres tomen ventaja en las prácticas que involucran este tipo de comunicación, debido su efecto emocionalmente positivo para ellas ${ }^{(13,14,15)}$.
Hall y colaboradores en un estudio comparativo por género, abordaron aspectos del trato de los médicos hombres y mujeres, así como de la satisfacción de sus pacientes, observando que las mujeres ejercen la comunicación con sus pacientes con mayor calidad, aun cuando los pacientes no refieren mayor satisfacción con su trato. Como explicación, los autores proponen que los pacientes esperan ser tratados con calidez por las mujeres médicos que, por sus pares del sexo masculino, por lo cual lo perciben como un aspecto implícito en el trato femenino y no genera en ellos una mayor satisfacción. En el trabajo liderado por Hall se exhibe la necesidad de valorar la mayor calidez en el trato de las médicas ${ }^{(15,16)}$.

Los hallazgos de este estudio podrán servir como un parámetro de comparación y punto de partida para nuevos estudios que pretendan esclarecer las diferencias en el desempeño de los estudiantes de medicina, pero más allá de ello, nos indican que es importante reconocer y fomentar la importancia del trato interpersonal para mantener la empatía entre las médicas a lo largo de toda su vida académica y profesional e inculcar a los médicos que estas competencias son importantes, no solo entre sus pares del sexo femenino, sino una parte fundamental de la relación médico-paciente. Así, como comclusión, se ha demostrado un mejor desempeño en la práctica de flebotomía entre los estudiantes de medicina del sexo femenino, principalmente derivada de los rubros que implican el trato interpersonal.

\section{REFERENCIAS BIBLIOGRÁFICAS}

1. Guldbrand D, Moercke N, Mette A, WickmannHansen G, Eika B. Skills Training in Laboratory and Clerkship: Connections, Similarities, and Differences. Med Educ Online. 2003;8(12).

2. McKenzie F. Medical Simulation: Utilize, Inspire, Embrace, Verify. Int J Med Surg. 2014;1(1):3-4.

3. Unterman A, Achiron A, Gat I, Tavor O, Ziv A. A novel simulation-based training program to improve clinical teaching and mentoring skills. Isr Med Assoc J. 2014;16(3):184-90.

4. Cheng A, Grant V, Dieckmann P, Arora S, Robinson T, Eppich W. Faculty development for simulation programs: Five issues for the future of debriefing training. Simul Healthc. 2015;10(4):217-22. https:// doi.org/10.1097/SIH.0000000000000090

5. Luna-Villanueva E, Santos-Rodríguez Mdl, Sierra Basto G, González-Arriaga CR, Zamora-Graniel FG. Retroalimentación integral (debriefing) oral y asistida por video en simulación de reanimación cardiopulmonar avanzada: estudio piloto. Fundación Educación Médica. 2015;18(2):139-47.

6. McCausland L, Curran C, Cataldi P. Use of a human simulator for undergraduate nurse education. Int J Nurs Educ Scholarsh. 2004;1:Article23.

7. Chen D, Lew R, Hershman W, Orlander J. A Cross-sectional Measurement of Medical Student Empathy. J Gen Intern Med. 2007;22(10):1434-8.

Tabla 4. Regresión lineal del puntaje obtenido y los factores asociados a los alumnos N=138.

\begin{tabular}{|c|c|c|c|c|c|c|}
\hline Dependiente & Independientes & Coeficiente & Error Estándar & $\mathrm{p}$ & \multicolumn{2}{|c|}{ Intervalo Confianza 95\% } \\
\hline \multirow[t]{3}{*}{ TEÓRICO } & Masculino & $-0,93$ & 1,26 & 0,461 & $-3,43$ & 1,57 \\
\hline & Edad & 0,01 & 0,93 & 0,995 & $-1,84$ & 1,85 \\
\hline & Grupo & 0,16 & 0,57 & 0,775 & $-0,97$ & 1,29 \\
\hline \multirow[t]{3}{*}{ PRÁCTICO } & Masculino & $-3,41$ & 1,46 & 0,021 & $-6,30$ & $-0,52 *$ \\
\hline & Edad & $-0,72$ & 1,07 & 0,506 & $-2,84$ & 1,41 \\
\hline & Grupo & 1,21 & 0,66 & 0,07 & $-0,10$ & 2,52 \\
\hline \multirow[t]{3}{*}{ PROMEDIO } & Masculino & $-2,17$ & 1,03 & 0,037 & $-4,21$ & $-0,13 *$ \\
\hline & Edad & $-0,34$ & 0,76 & 0,651 & $-1,85$ & 1,16 \\
\hline & Grupo & 0,62 & 0,47 & 0,188 & $-0,31$ & 1,54 \\
\hline
\end{tabular}


https://doi.org/10.2147/AMEP.S76800

8. Quince T, Thiemann P, Benson J, Hyde S. Undergraduate medical students' empathy: current perspectives. Adv Med Educ Pract. 2016;7:443-55. https://doi.org/10.2147/AMEP.S76800

9. Ren GSG, Min JTY, Ping YS, Shing LS, Win MTM, Chuan HS, et al. Complex and novel determinants of empathy change in medical students. Korean J Med Educ. 2016;28(1):67-78. https://doi.org/10.3946/ kjme.2016.11

10. Jagsi R, Griffith KA, DeCastro RA, Ubel P. Sex, role models, and specialty choices among graduates of US medical schools in 2006-2008. J Am Coll Surg. 2014;218(3):345-52. https://doi.org/10.1016/j. jamcollsurg.2013.11.012

11. Saleh J. Are Men from Mars and Women from Venus?: Bridging the gender learning gap in medical education. Sultan Qaboos Univ Med J. 2016;16(3):e267. 10.18295/squmj.2016.16.03.001

12. Kaatz A, Carnes M. Stuck in the out-group: Jennifer can't grow up, Jane's invisible, and Janet's over the hill. J Womens Health. 2014;23(6):481-4. https://doi. org/10.1089/jwh.2014.4766

13. Moffat KJ, McConnachie A, Ross S, Morrison JM. First year medical student stress and coping in a problem-based learning medical curriculum. Med Educ. 2004;38(5):482-91. https://doi.org/10.1046/ j.1365-2929.2004.01814.x
14. Sheehy AM, Kolehmainen C, Carnes M. We specialize in change leadership: A call for hospitalists to lead the quest for workforce gender equity. $\mathrm{J}$ Hosp Med. 2015;10(8):551-2. https://doi.org/10.1002/ jhm.2399

15. Hall JA, Roter DL, Blanch-Hartigan D, Mast MS, Pitegoff CA. How patient-centered do female physicians need to be? analogue patients' satisfaction with male and female physicians' identical behaviors. Health Commun. 2015;30(9):894-900. https://doi.org/10.1 080/10410236.2014.900892

16. Russell RS, Johnson DM, White SW. Patient perceptions of quality: analyzing patient satisfaction surveys. Int J Oper Prod Man. 2015;35(8):1158-81. 